\title{
Soft tissue augmentation of ridge defects in the maxillary anterior area using two different methods: a randomized controlled clinical trial
}

\author{
Akcalı, A ; Schneider, D ; Ünlü, F ; Bıcakcı, N ; Köse, T ; Hämmerle, C H F
}

\begin{abstract}
OBJECTIVES: To test whether or not vascularized interpositional periosteal-connective tissue grafts are as successful as free subepithelial connective tissue grafts in augmenting volume defects in the anterior maxilla. MATERIAL AND METHODS: Twenty subjects with Seibert class 1 ridge defects in the anterior maxilla were randomly, equally assigned to augmentation by vascularized interpositional periosteal-connective tissue graft (test) or free subepithelial connective tissue graft (control). Clinical periodontal parameters at teeth adjacent to the gap were recorded, and conventional impressions were taken prior to surgery (baseline = t0 ) and $1(\mathrm{t} 1), 3$ (t3) and 6 (t6 ) months after surgery. The casts were optically scanned, digitized and analyzed for ridge contour changes in the augmented area. Data were subjected to nonparametric statistics. RESULTS: The contour changes in labial distance between baseline and follow-up for the control group were (median, range) $1 \mathrm{~mm}, 0.37-1.45$ ( $\mathrm{t} 0$ -t1 ); $1.18 \mathrm{~mm}, 0.39-1.40$ (t0 -t3 ); and 0.63 mm, 0.28-1.22 (t0 -t6 ) and for test group $1.21 \mathrm{~mm}, 0.74-2.47$ (t0 -t1 ); $1.26 \mathrm{~mm}, 0.50-1.71$ (t0 -t3 ); and $1.18 \mathrm{~mm}, 0.16-1.75$ (t0 -t6 ). Significantly less shrinkage of the graft was observed in the test group after 6 months $(\mathrm{P}=0.03)$. Clinical periodontal parameters at the neighboring teeth were stable over the follow-up period and did not differ between groups. CONCLUSIONS: Augmentation of single tooth gaps with moderate ridge defects in the anterior maxilla was successfully performed using both techniques. However, after 6 months, sites treated by the pediculated graft were superior in maintaining the initially augmented volume and showed less shrinkage of the graft. This could be attributed to better perfusion of the pediculated graft.
\end{abstract}

DOI: https://doi.org/10.1111/clr.12368

Posted at the Zurich Open Repository and Archive, University of Zurich

ZORA URL: https://doi.org/10.5167/uzh-96101

Journal Article

Accepted Version

Originally published at:

Akcalı, A; Schneider, D; Ünlü, F; Bıcakcı, N; Köse, T; Hämmerle, C H F (2015). Soft tissue augmentation of ridge defects in the maxillary anterior area using two different methods: a randomized controlled clinical trial. Clinical Oral Implants Research, 26(6):688-695.

DOI: https://doi.org/10.1111/clr.12368 
Title: Soft Tissue Augmentation of Ridge Defects in the Maxillary Anterior Area Using Two Different Methods: A Randomized Controlled Clinical Trial

Authors:

A AKCALI ${ }^{1}$, D SCHNEIDER ${ }^{2}$, F ÜNLÜ $^{1}, \mathrm{~N} \mathrm{BICAKCI}^{1}, \mathrm{~T} \mathrm{KÖSE}^{3}$, C. H. F. HÄMMERLE ${ }^{2}$

${ }^{1}$ Department of Periodontology, School of Dentistry, Ege University, İzmir, Turkey

${ }^{2}$ Clinical of Fixed and Removable Prosthodontics and Dental Material Science, Center of Dental Medicine, University of Zurich, Zurich, Switzerland

${ }^{3}$ Department of Biostatistics and Medical Informatics, Ege University, İzmir, Turkey

Running title: Soft Tissue Augmentation of Ridge Defects

Corresponding author:

Dr Aliye Akcal 1

Department of periodontology

School of dentistry,

Ege University

35100 Bornova, İzmir / Turkey

Tel: + 902323881105

Fax: + 902323880325

@: aliyeakcali@hotmail.com

Keywords: soft tissue augmentation, ridge defect, subepithelial connective tissue graft, volumetric measurement 


\begin{abstract}
:
Objectives: To test whether or not vascularized interpositional periosteal-connective tissue grafts are as successful as free subepithelial connective tissue grafts in augmenting volume defects in the anterior maxilla.
\end{abstract}

Material and Methods: 20 subjects with Seibert class 1 ridge defects in the anterior maxilla were randomly, equally assigned to augmentation by vascularized interpositional periostealconnective tissue graft (test) or free subepithelial connective tissue graft (control). Clinical periodontal parameters at teeth adjacent to the gap were recorded and conventional impressions were taken prior to surgery (baseline $=\mathrm{t} 0)$ and $1(\mathrm{t} 1), 3(\mathrm{t} 3)$ and $6(\mathrm{t} 6)$ months after surgery. The casts were optically scanned, digitized and analyzed for ridge contour changes in the augmented area. Data were subjected to non-parametric statistics.

Results: The contour changes in labial distance between baseline and follow-up for the control group were (median; range) 1mm; 0.37-1.45 (t0-t1), 1.18mm; 0.39-1.40 (t0-t3) and 0.63mm; 0.28-1.22 (t0-t6) and for test group 1.21mm; 0.74-2.47 (t0-t1), 1.26mm;0.50-1.71 (t0-t3) and $1.18 \mathrm{~mm} ; 0.16-1.75$ (t0-t6). Significantly less shrinkage of the graft was observed in the test group after 6 months $(\mathrm{p}=0.03)$. Clinical periodontal parameters at the neighboring teeth were stable over the follow-up period and did not differ between groups.

Conclusions: Augmentation of single tooth gaps with moderate ridge defects in the anterior maxilla was successfully performed using both techniques. However, after 6 months, sites treated by the pediculated graft were superior in maintaining the initially augmented volume and showed less shrinkage of the graft. This could be attributed to better perfusion of the pediculated graft. 


\section{Introduction:}

Several factors such as tooth loss, periodontal diseases, trauma, and congenital diseases can cause ridge defects (Fiorellini \& Nevins 2003; Gasparini 2004). These defects often need to be augmented for an optimal prosthetic restoration with respect to esthetics, phonetics and hygiene (Seibert 1983a). Soft tissue augmentation procedures have widely been used for surgical correction of localized alveolar ridge defects, for pre-prosthetic site development and for ridge preservation (Seibert \& Salama 1996; Studer et al. 2000). Several methods using autogenous grafts have been described for pontic site development in partially edentulous patients (Pini Prato. 2004, Thoma et al. 2009). These techniques include subepithelial connective tissue grafts (Garber \& Rosenberg 1981), onlay grafts (Seibert 1983b), connective tissue pedicle grafts (Scharf \& Tarnow 1992) and their modifications (Sclar 2003; Kim et al. 2012). Among these techniques, subepithelial connective tissue grafts and free gingival grafts are often used and lead to successful clinical results (Langer et al. 1980; Abrams et al. 1980). Free subepithelial connective tissue grafts are generally preferred over free gingival grafts especially in areas with high esthetic impact due to their advantages regarding the gain of tissue volume as well as better color and texture match (Edel et al. 1974; Nemcovsky et al. 2000). Even though free connective tissue grafts are widely used for soft tissue augmentation, it has previously been reported that an important disadvantage of these procedures was the significant shrinkage of the grafts following the augmentation procedure (Mörmann et al. 1981; Studer et al. 2000; Thoma et al. 2010). In order to overcome this disadvantage, pedicle connective grafts have been applied for ridge defects augmentation and successful clinical results have been reported (Abrams 1980; Scharf \& Tarnow 1992; Sclar 2003; Park et al. 2012). It has been speculated that pediculated connective tissue grafts result in less shrinkage because of the intact blood supply during the healing period (Sclar 2003). This hypothesis, however, has never been substantiated with studies comparing the long-term stability of free and pedicle connective tissue grafts. Although different established 
and newly developed materials and techniques for ridge augmentation have been tested using clinical protocols, there is still a lack of information about the stability of augmented tissues and applying these procedures. The aim of this randomized controlled clinical trial was, therefore, to test whether or not vascularized interpositional periosteal-connective tissue grafts (VIP-CTG) are as successful as free subepithelial connective tissue grafts (CTG) in augmenting volume defects of the alveolar ridge in the maxillary anterior area and in maintaining the volume augmented. 


\section{Material and Methods:}

\section{Study design}

This study was designed as a randomized, controlled clinical trial and was conducted at Ege University, School of Dentistry, Department of Periodontology, İzmir, Turkey between January 2010 and September 2011. Volumetric evaluation was performed at the University of Zurich, Center of Dental Medicine, Department of Fixed and Removable Prosthodontics and Dental Material Science, Zurich, Switzerland. Twenty patients with single tooth gaps in the maxillary anterior area presenting Seibert Class I ridge defects were included in the study (Figure 1.a). The healing period after the tooth extraction had to be at least three months prior to the surgical procedures. To be included in the study, patients also had to be systemically and periodontally healthy non-smokers. Patients with general contraindications against surgical procedures were excluded. The study was conducted in accordance with ethical principles, including the World Medical Association Declaration of Helsinki. The study protocol was explained to the patients and written informed consent was received from each individual before participation. The protocol of the study was approved by the Ethics Committee of Faculty of Medicine, Ege University (09-5.1/17; Protocol code: 2009/DIS/008). Data were recorded within a month prior to surgery (baseline) and 1, 3 and 6 months postoperatively (Figure 2).

\section{Clinical periodontal measurements}

Patients were initially examined. All patients received oral hygiene instructions and non-surgical periodontal treatment was performed if necessary. At baseline, clinical periodontal measurements including plaque control record (O’Leary et al. 1972), bleeding on probing (BOP) (Ainamo \& Bay 1975), probing pocket depth (PPD) and clinical attachment level (CAL) were recorded at teeth adjacent to the gap using a Williams periodontal probe (Hu-Friedy, Chicago, IL).

Impressions were taken using high viscosity silicon impression material (GC Exaflex, GC Corporation). Models were cast in dental stone and used as the baseline reference for volumetric 
measurements (GC Fujirock type 4, GC Corporation). One, three and six months after the surgical volume augmentation procedure, clinical periodontal measurements were recorded, impressions were retaken and models were cast.

\section{Surgical procedures}

Randomization was performed immediately before the surgery using computer-generated lists and subjects were assigned equally to one of two treatment groups: In the test group, a modified vascularized interpositional periosteal-connective tissue grafting procedure (VIP-CTG) was performed (Sclar 2003). In the control group, a free subepithelial connective tissue graft (CTG), harvested from the palate, was used (Garber \& Rosenberg 1981) (Figure 1).

In both groups, after local anesthesia (2\% lidocaine, 1:100.000 epinephrine), incisions were placed over the crest of the ridge reaching to alveolar bone (Figure 1.b). Papillae were included in the incision line only when there was a loss of papillary height. A deep supraperiosteal soft tissue pouch was prepared by sharp dissection extending apically to the mucogingival line and to the neighboring teeth mesio-distally in both groups (Figure 1.c). In the control group, the pouch was extended $2 \mathrm{~mm}$ to the palatal aspect of the defect area. Subsequently, a free subepithelial connective tissue graft measuring $5 \times 10 \mathrm{~mm}$ was harvested from the contralateral palate using the trap door approach (Edel 1974) (Figure 1.d, f). The donor site was closed by horizontal and single interrupted sutures 6-0 Polypropylene (Blue Monofilament, 13mm, 3/8 Circle, $45 \mathrm{~cm}$, Ethicon, USA). The free gingival graft was trimmed using a surgical blade to a dimension $5 \times 10$ millimeters and a thickness of 1.0 to 1.5 millimeters as measured by a ruler and a caliper. Subsequently, the graft was inserted into the pouch that was previously prepared at the recipient site (Figure 1.h) and secured with two non-resorbable sutures at the labial and the palatal aspect (Figure 1.i). The flap margins at the crest were adapted using single interrupted sutures (Figure $1 . j)$. 
In the test group, a pediculated subepithelial connective tissue graft also measuring $5 \times 10 \mathrm{~mm}$ was prepared at the ipsilateral palatal area. The pediculated connective tissue graft was undermined using a blade, a forceps and a small raspatorium, then mobilized and rotated into the pouch at the recipient site (Figure 1.e, g, h). It was secured by sutures in the same manner as in the control group (Figures 1.i, j). Wound closure was achieved as in the control group.

Patients were instructed to avoid mechanical cleaning in the area of surgery, to apply a cold pack during the first $6 \mathrm{~h}$ after the surgery and to rinse with a chlorhexidine $0.12 \%$ solution twice a day for 10 days. No antibiotics were prescribed. The pontic of the provisional partial removable prosthesis was adapted to avoid pressure on the augmented tissue. Sutures were removed after 10 days. After a 6-month healing period, final fixed prosthetic reconstructions were placed.

\section{Volumetric analysis to evaluate soft tissue volume changes}

In order to evaluate the volumetric changes of soft tissues between baseline and 6 months, all the stone models were digitized using a lab-based optical scanner (Imetric 3D GmbH, Courgenay, Switzerland). The digital surface models were imported as stl-files into the software for volumetric analysis (Swimmeda/SMOP, Zürich, Switzerland). Digital cast models representing the different time-points during the treatment were superimposed using the best-fit algorithm using tooth surfaces as references. The relevant area for the measurements of volume changes was defined according to previous studies on ridge contour alterations (Fickl et al. 2009; Thoma et al. 2010; Schneider et al. 2011). The relevant area was defined by the mesial and distal papillary midline, the mucogingival line, and the alveolar crest. Since this area was different in size for each site, the mean change of volume per area was calculated, resulting in a distance in labial direction. By doing so different sites could be directly compared in terms of volume changes irrespective of their size and the size of the measured area (Figure 3). Before the initiation of the study, the reproducibility of the volumetric measurement method was evaluated and an intraclass correlation coefficient of 0.91 was calculated for two consecutive measures. 


\section{Assessment of Pain}

Visual Analogue Scale (VAS) was used to evaluate the pain level during the $1^{\text {st }}$ week of postoperative healing period. A $100 \mathrm{~mm}$ horizontal linear scale with "none" at the left and "unbearable" at the right end as verbal end points was prepared. Forms were given to patients just after completion of the operations and they were asked to mark the pain value that they feel at the end of each day. Forms were collected on the day of suture removal and the distance of the mark to the very left end of the scale was measured using a ruler and noted as mm values.

\section{Statistical analysis}

Mean values and standard deviations were calculated for the clinical periodontal measurements. Soft tissue volume change values were generated including median values and range. Data obtained thereof were subjected to the nonparametric Friedman tests and confirmed between groups by Mann-Whitney $U$ test. The level of significance was set at $p<0.05$. The statistical analyses were performed using a statistical software package (SPSS version 17 Chicago, Illinois, USA). 


\section{Results:}

\section{Patient demographics}

During the study, all patients showed uneventful healing in the area of surgery. Intraoperative or postoperative complications including bleeding, necrosis and abnormal swelling occurred neither at the recipient nor at the donor sites. In the control group three patients had to be excluded due to different reasons during the follow-up period ( 2 were moving to another city and one patient's general health did not allow for follow-up visits). The demographics of the patients are provided in Table 1 and distributions of missing teeth are depicted in Table 2. Demographic variables and location of the treated edentulous areas were similar at the beginning of the study for both groups $(\mathrm{p}>0.05)$.

\section{Volumetric measurements}

The primary parameter in the volumetric analysis was the change in soft tissue volume in the area of tissue augmentation between baseline and 6 months. The selected area used for volumetric measurements was similar in both groups $(\mathrm{p}=0.20)$ (Table 3). The thickness of the connective tissue graft was measured in both groups. No statistically significant differences were observed between groups (test; mean: $1.41 \pm 0.08$ and control; mean: $1.4 \pm 0.08)(\mathrm{p}=0.93)$.

Volume gain after surgery, expressed as a distance in labial direction from calculation of volume per area, was documented in both treatment groups. In detail, at one month after surgery (t0-t1) a gain of $1.21 \mathrm{~mm}$; range $0.74-2.47$ was recorded in the test group and $1 \mathrm{~mm}$; range 0.37 1.45 in the control group. At three months, the soft tissue volume change (t0-t 3$)$ was similar in both groups and amounted to $1.26 \mathrm{~mm}$; range $0.50-1.71$ in the test group and $1.18 \mathrm{~mm}$; range 0.39-1.40 in the control group ( $\mathrm{p}=0.30$ ) (Table 3). At six months, the volumetric gain compared to baseline (t0-t6) was significantly higher in the pedicle group $(1.18 \mathrm{~mm}$; range $0.16-1.75)$ compared to the free connective tissue group $(0.63 \mathrm{~mm}$; range $0.28-1.22)(\mathrm{p}=0.03)$. At 6 months the control group had lost almost half of the volume gain recorded at 1 month. No statistically 
significant differences were observed between the pediculated and free connective tissue graft group $(\mathrm{p}=0.80)$ at 1 and 3 months (t0-t1 and t0-t 3$)$ (Table 3$)$.

The mean shrinkage in soft tissue volume between baseline and 6 months was statistically higher for the control group (47\%) compared with the test group (6.4\%) (t0-t6). Slightly higher volume gain was observed at 3 months in both groups without any statistically significant differences between the two treatments $(\mathrm{t} 0-\mathrm{t} 3)(\mathrm{p}>0.05)$.

\section{Clinical periodontal parameters and pain levels}

Clinical periodontal parameters including PCR, BOP, PPD and CAL were recorded at teeth adjacent to the defect area and are displayed in table 4. Changes of these parameters were recorded in both groups during the treatment, however, PPD and CAL parameters remained within the limits considered healthy during the observation period in both groups. Pain levels showed no statistically significant differences between the study groups at any time point $(\mathrm{p}>0.05)$. 


\section{Discussion:}

The results of the present study demonstrated both free and pediculated connective tissue grafts to lead to successful soft tissue volume augmentation in Seibert Class I ridge defects. The median contour gain in labial direction at the 3-months follow-up measured $1.3 \mathrm{~mm}$ in the test and $1.2 \mathrm{~mm}$ in the control group. The VIP-CTG (test group) was advantageous with respect to maintaining the augmented volume during the entire 6-months observation period compared to the free CTG. Between 3 and 6 months, a mean shrinkage of almost half of the volume (47\%) occurred in the control group while most of the augmented tissue remained stable in the test group (mean shrinkage $6.4 \%$ ). The treatment modality did not have an impact on periodontal health at adjacent teeth. Both techniques were equally tolerated by the patients in terms of postoperative discomfort.

The augmentation of alveolar ridge defects can be indicated in various clinical situations to improve esthetics, function, hygiene and long-term tissue stability. In general, a lack of tissue can be reconstructed either at the bone level by using bone augmentation procedures (Hammerle et al. 2008) or at the soft tissue level by the use of connective tissue grafts (Thoma et al. 2009). Usually, bone augmentation procedures are performed in connection with implant site development or for the reconstruction of extensive ridge defects. However, for the development of pontic areas, especially ovate pontics, sufficient soft tissue thickness is necessary allowing for conditioning the pontic area (Abrams 1980; Garber \& Rosenberg 1981). Creating an appropriate soft tissue condition and pontic design improves function, esthetics and cleansibility (Calesini et al. 2008).

The augmentation of ridge defects with soft tissue grafts is a well-documented approach in mucogingival surgery. Results of pre-clinical and clinical studies have been shown different techniques to be successful (Langer et al. 1980; Abrams 1980; Seibert 1983b; Seibert \& Louis 1996; Kim et al. 2012; Zucchelli et al. 2013). Despite their successful clinical application, there 
is little knowledge regarding the long-term behavior of the grafts in the augmented area in terms of volume stability.

In a recent clinical study, the stability of the augmented peri-implant tissues was measured during one year following bone and subepithelial connective tissue grafting (Schneider et al. 2011). The same techniques as in the present study were applied for assessing the volume changes over time. Only minimal changes $(0.04 \pm 0.31 \mathrm{~mm})$ were observed in terms of soft tissue contour reduction at the labial aspect of the augmented area between the post-surgical and the one-year follow-up examinations (Schneider et al. 2011). The investigators concluded that the clinical methods applied were associated with a high degree of stability of the augmented tissue volume. The results of that investigation, however, cannot directly be compared to studies on edentulous areas without implant insertion and bone augmentation.

A recent animal study in dogs assessed the effect of soft tissue augmentation at edentulous sites without implant placement comparing different methods and materials (Thoma et al. 2010). Sham-operated control sites were compared with autogenous subepithelial connective tissue grafts and a newly developed collagen matrix. Volume changes of the grafted areas were followed for 28 and 84 days using the same method for evaluation of contour changes as in the present study. The CTG resulted in mean volume gain in buccal direction of $1.5 \mathrm{~mm}$ at 28 days and $1.4 \mathrm{~mm}$ at 84 days. Similar changes were observed in the collagen matrix group (1.6 $\mathrm{mm}$ and $1.4 \mathrm{~mm}$ respectively), whereas the control sites showed a slight mean decrease in contour from 0.0 at 28 days to -0.3 at 84 days $(-0.3 \mathrm{~mm})$. In the present study a similar amount of mean tissue gain was observed as compared to the above-mentioned animal experiment. Between the post-augmentation and the three-month observation stability of the augmented volume was recorded in the present study. At six months, however, the CTG group demonstrated a significant loss of the augmented volume. The augmented volume in the test group in contrast had remained stable between 3 and 6 months. Direct comparison between the two studies is 
difficult due to the different designs (animal vs human, size and location of defects) and followup periods. No comparative data on volume changes over 6-months periods are currently available from other investigations.

Only very few clinical studies are available on soft tissue augmentation in pontic areas (Allen 1985, Studer et al. 2000). Only one recent three-armed study compared free connective tissue grafts including an epithelium layer (FGG) and free subepithelial connective tissue grafts (CTG) and untreated sites (Studer 2000). Cast models from impressions obtained before treatment and after an observation period of 1 and 3.5 months were analyzed for volumetric changes using the Moiree method (Studer et al. 1997). The CTG group showed more volume gain after augmentation than the FGG at both follow-up time points. After one month, the mean volume gain was $187 \mathrm{~mm}^{3}$ in the CTG and $117 \mathrm{~mm}^{3}$ in the FGG group. At 3.5 months, a decrease of the soft tissue volume of $15 \%$ occurred in the CTG group and $11.1 \%$ in the FGG group. This difference of only $4 \%$ between groups can be considered clinically insignificant.

In the present study, a high degree of volume stability and even a slight volume gain was observed between one month and three months in both treatment groups. This is in contrast to other studies reporting the tissue volume changes to occur mostly during the initial phase of the wound healing process following the augmentation procedures (Studer et al. 2000, Lindhe et al. 2008). Possibly, the defect morphology and location within the dental arch, the graft composition, the surgical technique, and the provisional prosthetic rehabilitation could have an influence on the tissue healing and volumetric alterations at the recipient site. Also, a higher number of patients with an even distribution between treatment groups would provide more solid data in future investigations. In the present study, the graft shrinkage, as mentioned in the other investigations (Studer et al. 2000, Lindhe et al. 2008), occurred only between 3 months to 6 months. This finding is somehow surprising, since the most pronounced alterations were expected to take place within the first 3 months. However, available clinical studies reported on 
shorter-term data and did not include observation periods of the same length as in the present investigation. It has been recommended not to initiate final restorative measures until 6 months after ridge augmentation procedures, since qualitative and possibly quantitative alterations can ocur during this period due to maturation (Lindhe et al. 2008). This statement is supported by the findings in the present study. However, it is possible that conditioning of the augmented area using pontics would again influence the tissue behavior and lead to different results. This, again, should be addressed in future research.

The difference in loss of volume between groups in the present study could be explained by a better blood perfusion in the test group. This may lead improved graft integration and less soft tissue resorption compared to the control group. No vertical incision was performed at the recipient area in order to allow for better blood supply from the lateral direction in addition to the pedicle part. It must be recognized, however, that the test group showed remarkable variability in soft tissue volume change at three and especially six months leading to a large standard deviation. A possible explanation could be the technically more demanding surgical procedure, the variation in graft quality and the individually different perfusion of the pediculated graft. In the control group, a free connective tissue grafting procedure was performed (Garber \& Rosenberg 1981). In this procedure, the initial incision at the recipient site may be made in various ways such as apico-coronally or mesio-distally. Performing the incision mesio-distally on the crest of the ridge without additional incisions, as it was done in the control group, offers the advantages of minimizing the risk for perforations close to the defect area and of keeping the blood supply from the surrounding buccal tissues intact.

Another aspect regarding tissue stability could be the graft thickness. In the present study, the thickness of the connective tissue graft was measured in four areas using a caliper and was kept identical in both groups to investigate the influence of the pedicle rather than the thickness of the 
graft. However, the additional influence of the thickness and also possibly the quality or histological composition of the graft would be of great interest for future research.

Since different methods and parameters were being used for the evaluation of soft tissue augmentation procedures, it is difficult to compare the outcome of the published studies (Thoma et al. 2009; Ronay et al. 2011). A novel approach for the measurement of contour changes has recently been introduced and applied in different studies including the present one (Windisch et al. 2007; Fickl et al. 2009; Strebel 2009; Thoma et al. 2010; Schneider et al. 2011). In this novel method impressions of the clinical areas under investigation are being taken. These impressions are subsequently transformed into cast stone models. These models are then optically scanned and these digital scans are further used for analysis. A particular software program allows linear and morphological assessments of the area under investigation. This software further allows superimposing different digital scans for assessing differences in linear and volumetric data between various clinical time points and situations. This method allows for highly accurate measurement in the region of interest. However, the accuracy of this method is especially influenced by the quality of the impression and of the cast fabrication as well as the presence of stable reference points or surfaces. Advantages of this method include the possibilities for linear and volumetric measurements, comparison of different clinical situations and time points, comparison of data from different studies, easy storage of the data, transfer of the data via the Internet, assessments of large samples demanding little resources and costs. All these technical and handling benefits will hopefully allow this method to be more widely applied in future studies thus allowing to gather sound comparative data on the clinical effects of different volume augmentation procedures. 


\section{Conclusion}

Within the limitations of this study it may be concluded that both soft tissue augmentation techniques were effective in increasing the volume of defective alveolar ridges in single tooth gaps in the anterior maxilla. However, possibly due to better perfusion, the pediculated connective tissue resulted in less shrinkage of the graft during the 6-months healing period. Neither of the two techniques had an effect on clinical periodontal parameters at the adjacent teeth during the follow-up period. Also, no difference was observed in postoperative pain. Further studies with larger patient populations are needed to verify the present findings and to evaluate soft tissue gain by different soft tissue augmentation techniques. 


\section{Acknowledgements:}

This study was supported by the grant from the Ege University Research Foundation (2009/DIS/008), İzmir, Turkey. The authors would like to express their thanks to Swissmeda AG, Zürich, Switzerland, for providing technical support in the volumetric analysis.

The authors declare that they have no conflict of interest. 


\section{References:}

1 Abrams, L. (1980) Augmentation of the deformed residual edentulous ridge for fixed prosthesis. Compendium of Continuing Education in Dentistry 1: 205-213.

2 Allen, E.P., Gainza C.S., Farthing G.G., Newbold D.A. (1985) Improved technique for localized ridge augmentation. A report of 21 cases. Journal of Periodontology 56: 195-199.

3 Ainamo, J, Bay, I. (1975) Problems and proposals for recording gingivitis and plaque. International Dental Journal 25: 229-235.

4 Calesini G, Micarelli C, Coppè S, Scipioni A. (2008) Edentulous site enhancement: a regenerative approach for the management of edentulous areas. Part 1. Pontic areas. The International Journal of Periodontics and Restorative Dentistry 28: 517-523.

5 Edel, A. (1974) Clinical evaluation of free connective tissue grafts used to increase the width of keratinized gingiva. Journal of Clinical Periodontology 1: 185-196.

6 Fickl, S., Schneider, D., Zuhr, O., et al. (2009) Dimensional changes of the ridge contour after socket preservation and buccal overbuilding: an animal study. Journal of Clinical Periodontology 36: 442-448.

7 Fiorellini, J.P., Nevins, M.L. (2003) Localized ridge augmentation/preservation. A systematic review. Annals of Periodontology 8: 321-327.

8 Garber, D.A., Rosenberg, E.S. (1981) The edentulous ridge in fixed prosthodontics. Compendium of Continuing Education in Dentistry 2: 212-223.

9 Gasparini, D.O. (2004) Double-fold connective tissue pedicle graft: a novel approach for ridge augmentation. The International Journal of Periodontics and Restorative Dentistry 24: 280-287.

10 Hämmerle CH, Jung RE, Yaman D, Lang NP. (2008) Ridge augmentation by applying bioresorbable membranes and deproteinized bovine bone mineral: a report of twelve consecutive cases. Clinical Oral Implants Research 19: 19-25. 
11 Kim, C.S., Jang, Y.J., Choi, S.H., et al. (2012) Long-term results from soft and hard tissue augmentation by a modified vascularized interpositional periosteal-connective tissue technique in the maxillary anterior region. Journal of Oral and Maxillofacial Surgery 70: 484-491.

12 Langer, B, Calagna, L. (1980) The subepithelial connective tissue graft. The Journal of Prosthetic Dentistry 44: 363-367.

13 Lindhe, J., Karring, T., Araujo, M. (2008) The anatomy of periodontal tissues. In: Lindhe J., Karring T., Lang N.P. Clinical periodontology and implant dentistry. 5. Edition, p. 1020. Mungsgaard, Oxford.

14 Mörmann, W., Schaer, F., Firestone, A.R. (1981) The relationship between success of free gingival grafts and transplant thickness. Revascularization and shrinkage-a one year clinical study. Journal of Periodontology 52: 74-80.

15 Nemcovsky CE, Artzi Z, Moses O. (2000) Rotated palatal flap in immediate implant procedures. Clinical evaluation of 26 consecutive cases. Clinical Oral Implants Research 11: 83-90.

16 O'Leary, T.J., Drake, R.B., Naylor, J.E. (1972) The plaque control record. Journal of Periodontology 43: 38 .

17 Park, S.H., Wang, H.L. (2012) Pouch roll technique for implant soft tissue augmentation: a variation of the modified roll technique. The International Journal of Periodontics and Restorative Dentistry 32: 116-121.

18 Prato, G.P. (2004) Prevention of Alveolar Ridge Deformities and Reconstruction of Lost Anatomy: A Review of Surgical Approaches. The International Journal of Periodontics and Restorative Dentistry 24: 434-445. 
19 Ronay, V, Sahrmann, P, Bindl, A, Attin, T, Schmidlin, P.R. (2011) Current status and perspectives of mucogingival soft tissue measurement methods. Journal of Esthetic and Restorative Dentistry 23: 146-156.

20 Scharf, D.R, Tarnow, D.P. (1992) Modified roll technique for localized alveolar ridge augmentation. The International Journal of Periodontics and Restorative Dentistry 12: 415425.

21 Schneider, D., Grunder, U., Ender, A., et al. (2011) Volume gain and stability of peri-implant tissue following bone and soft tissue augmentation: 1-year results from a prospective cohort study Clinical Oral Implants Research 22: 28-37.

22 Sclar, A.G. (2003) Soft Tissue and Esthetic Considerations in Implant Therapy Quintessence $205-221$.

23 Seibert J.S, Salama, H. (1996) Alveolar ridge preservation and reconstruction. Periodontology 2000 11: 69-84.

24 Seibert, J.S. (1983a) Reconstruction of deformed, partially edentulous ridges, using full thickness onlay grafts. Part I. Technique and wound healing. Compendium of Continuing Education in Dentistry 4: 437-453.

25 Seibert, J.S. (1983b) Reconstruction of deformed, partially edentulous ridges, using full thickness onlay grafts. Part II. Prosthetic/periodontal interrelationships. Compendium of Continuing Education in Dentistry 4: 549-562.

26 Strebel, J., Ender, A., Paqué, F., Krähenmann, M., Attin, T., Schmidlin, P.R. (2009) In vivo validation of a three-dimensional optical method to document volumetric soft tissue changes of the interdental papilla. Journal of Periodontology 80: 56-61.

27 Studer S.P, Sourlier D., Wegmann U., Schärer P., Rees T.D. (1997) Quantitative measurement of volume changes induced by oral plastic surgery: validation of an optical 
method using different geometrically-formed specimens. Journal of Periodontology 68: 950962.

28 Studer, S.P., Lehner, C., Bucher, A., Schärer, P. (2000) Soft tissue correction of a single-tooth pontic space: a comparative quantitative volume assessment. The Journal of Prosthetic Dentistry 83: 402-411.

29 Thoma, D.S., Benić, G.I., Zwahlen, M., et al. (2009) A systematic review assessing soft tissue augmentation techniques. Clinical Oral Implants Research 4: 146-165.

30 Thoma, D.S., Jung, R.E., Schneider, D., et al. (2010) Soft tissue volume augmentation by the use of collagen-based matrices: a volumetric analysis. Journal of Clinical Periodontology 37: 659-666.

31 Windisch, S.I., Jung, R.E., Sailer, I, et al. (2007) A new optical method to evaluate threedimensional volume changes of alveolar contours: a methodological in vitro study. Clinical Oral Implants Research 18: 545-551.

32 Zucchelli G, Mazzotti C, Mounssif I, Marzadori M, Stefanini M. (2013) Esthetic Treatment of Peri-implant Soft Tissue Defects: A Case Report of a Modified Surgical-Prosthetic Approach. The International Journal of Periodontics and Restorative Dentistry 33:327-335. 


\section{Figures and Figure Legends:}

Figure 1: Surgical procedures

Figure 2: Study flow chart depicted treatment sequences and time points of evaluation

Figure 3: Volumetric measurement of soft tissues

\section{Tables and Table Legends:}

Table 1: Patient demographics

Table 2: Distributions of missing teeth

Table 3: Defect area values $\left(\mathrm{mm}^{2}\right)$ and thickness of the connective tissue graft $(\mathrm{mm})$

Table 4: Volumetric changes expressed as mean distance (millimeters) in labial direction in the measured area between evaluated time points Baseline (t0), 1 month ( $\mathrm{t} 1), 3$ months ( $\mathrm{t} 3), 6$ months (t6)

Table 5: Clinical periodontal parameters 